\section{Balls and feathers}

New J.Phys. (in the press); preprint at http://arxiv.org/pdf/1503.01213 (2015)

One of the first scientific tests of gravity was Galileo Galilei's experiment, in which he allegedly dropped balls of different masses from the Leaning Tower of Pisa. The idea that inertial and gravitational masses are identical was more rigorously formulated three hundred years later by Albert Einstein, as the equivalence principle. The universality of free fall that in a gravitational field, in the absence of friction, two bodies of different masses will drop at the same rate - would be taken for granted by most people, but even tiny violations can provide clues for quantum gravity models.

A modern take on Galileo's experiment uses matter-wave interferometry instead of balls and feathers. Jonas Hartwig and colleagues have proposed an experiment involving the preparation of an ultracold rubidium and ytterbium mixture in a magneto-optical trap, which is then released into a ten-metre free fall. They analysed the experimental conditions and found that in this atomic fountain, a new level of accuracy for ground tests can be reached - providing tighter bounds on the violation scenarios.

\section{To pinch a cloud}

Nature http://doi.org/10.1038/nature14291 (2015)

What is the role of ambient magnetic fields in star formation? Large-scale magnetic fields pervade the inter-cloud medium from which a giant molecular cloud gathers. These clouds, usually filamentary in shape, then form cores that eventually become stars. To investigate whether the magnetic field is dynamically important, Hua-bai Li and co-workers create magnetic field maps of NGC 6334 (the Cat's Paw nebula in Scorpius) from optical polarimetry data collected by several detectors, covering 100-0.01 parsec scales. The technique works because the polarization of starlight is parallel to the magnetic field, and for close-enough sources, the orientation is preserved.

According to their field maps, the main cloud filament lies perpendicular to the ambient field. Moreover, where the gas column density peaks, the magnetic field lines are 'pinched' together to form an hourglassshape perpendicular to the elongation direction. This anisotropic contraction shows that the magnetic field plays an important role in cloud fragmentation in NGC 6334, which is the nearest star-forming region producing massive stars (of more than 8 solar masses).

\section{Quantum biometrology}

Phys. Rev. E 91, 022714 (2015)

The absorption of light is responsible for triggering many biological sensing and regulation processes, including vision, photosynthesis and magnetoreception - the ability of some living organisms to detect the magnitude and orientation of the Earth's magnetic field, thought to be important for migration. These phenomena share common features, such as the excitation of a photosensitive molecule within a pigment-protein complex, which in turn sets off a series of chemical reactions leading to biological functionality. However, the fundamental mechanisms at play are unclear.

Now, a theoretical framework for understanding photoactivated biological processes as quantum measurements has been proposed. Borrowing ideas from

\title{
On the interface of it
}

Exciting physics often happens at interfaces. This can be taken quite literally in the case of surface plasmon polaritons - electromagnetic waves that can be excited at the interface between a metal and a dielectric, for example by irradiating with light. Direct observations of these quasiparticles are difficult to realize. But now, Luca Piazza and colleagues have found a way to both visualize the polaritons and control their spatial interference patterns.

The authors shot a femtosecond laser pulse at a silver nanowire suspended on a graphene film that was placed in a transmission electron microscope. The nanowire acted as a plasmonic nanoantenna, exciting surface plasmon polaritons that travelled back and forth and generated a standing light wave. Spatially resolving the electrons' energy losses (due to their interaction with the polaritons) led to images of the standing wave. Piazza etal. were able to show that by varying the polarization of the laser light, they could tune the nodal structure of the wave - capturing both the wave and particle nature of the polaritons.

magnetometry techniques, in which electron spins can act as quantum meters to sense weak magnetic fields, Atac Imamoglu and K. Birgitta Whaley have suggested that the pigment-protein complexes may act as quantum meters of incident radiation. In doing so, they have established when quantum coherence is essential for ensuring the biological functionality of a given photoactivated process, and when it is not. The authors identified magnetoreception as one example in which quantum coherent dynamics are crucial, providing experimentalists with a testable hypothesis for a largely confounding effect.

\section{Head first}

Proc. Natl Acad. Sci USA http://doi.org/22g (2015)

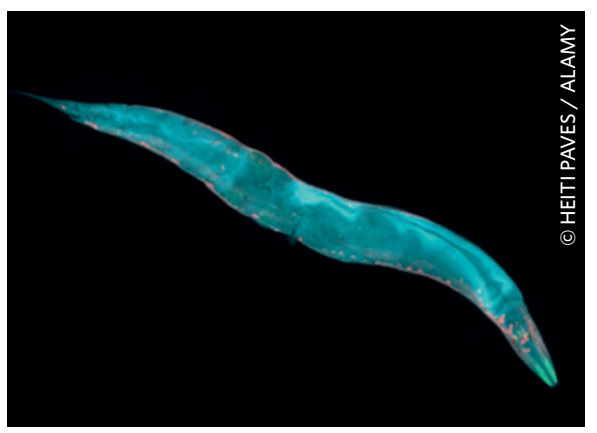

What's the best way of dealing with an oncoming current? For worms, and other undulatory aquatic animals, the answer might be to meet it head on. There is evidence to suggest that these low-Reynoldsnumber swimmers undergo rheotaxis, orienting themselves with or against a flow sometimes even overcoming chemical cues to do so. A study from Jinzhou Yuan and co-workers indicates that this may not be a conscious decision, and that rheotaxis could simply be a matter of hydrodynamics.

Yuan et al. performed experiments on swimming Caenorhabditis elegans (pictured) and noticed that near surfaces, the worms tended to orient themselves to face the flow. The team had previously identified interactions between the swimmer's flow field and the surface, which generated a torque that tilted the swimmer towards the surface. Experiments with touch-insensitive mutants confirmed that this was a hydrodynamic effect, independent of the animal's nervous system. Worms swimming to the surface were then subject to a velocity gradient: the fluid was stationary at the surface and so the animal's tail felt a stronger flow than its head, effecting a head-first orientation.

Written by May Chiao, Iulia Georgescu,

Abigail Klopper, Andrea Taroni and Bart Verberck. 